\title{
Infiltration of bupivacaine into the preperitoneal space and trocar incisions of patients undergoing laparoscopic totally extraperitoneal repair of unilateral inguinal hernia: a prospective randomized controlled observational study
}

\author{
Şükrü Çolak, Önder Akkuş, Bünyamin Gürbulak, Ekrem Çakar, Hasan Bektaş \\ Department of General Surgery, Istanbul Training and Research Hospital, Istanbul, Turkey
}

Videosurgery Miniinv 2020; 15 (1): 11-17

DOI: https://doi.org/10.5114/wiitm.2019.84385

\begin{abstract}
Introduction: Although laparoscopic repair of inguinal hernia is associated with reduced postoperative pain, it is not entirely painless. In addition to reducing the need for analgesic medication, postoperative complications, and hospitalization, postoperative pain control enables early return to normal activity.

Aim: To evaluate the efficacy of bupivacaine instilled into the pre-peritoneal space and trocar incisions of patients undergoing laparoscopic totally extraperitoneal (TEP) repair of inguinal hernia.

Material and methods: Bupivacaine was instilled into the pre-peritoneal space and trocar incisions of the patients in group I $(n=23)$, whereas it was infiltrated only into the trocar incisions of the patients in group II $(n=21)$. No local anesthetic was administered to the patients in group III $(n=21)$. Postoperative pain was assessed using the Visual Analog Scale (VAS) at 4 and $24 \mathrm{~h}$, and the dosage of analgesic medication was noted.

Results: No significant difference regarding age, gender, body mass index, ASA class, history of abdominal surgery, or smoking was noted between the three groups $(p>0.05)$. VAS score at $4 \mathrm{~h}$ was significantly higher in group III than in groups I and II ( $p<0.05)$. The dosage of analgesic medication was significantly higher in group III than in groups I and II ( $p<0.05)$, with no significant difference between groups I and II ( $p>0.05)$.

Conclusions: Infiltration of long-acting local anesthetic into the pre-peritoneal space and trocar incisions of patients undergoing laparoscopic TEP repair of inguinal hernia reduces the need for analgesic medication by reducing early postoperative pain.
\end{abstract}

Key words: Visual Analog Scale, bupivacaine, laparoscopic totally extraperitoneal.

\section{Introduction}

Repair of inguinal hernia is one of the most common operations performed in general surgery clinics. Regardless of the etiology and type, surgical repair is considered to be the definitive treatment for inguinal hernia [1]. Every year, more than 20 million repairs of inguinal and femoral hernias are performed worldwide [2]. Studies have revealed that there is an increase in the number of patients opting for laparoscopic repair of inguinal hernia, and the need for analgesic medication has drastically decreased because the procedure reduces postoperative pain [3-5].

The preperitoneal space and trocar incisions are the two potential pain sites in the laparoscopic totally extraperitoneal (TEP) procedure. Previous randomized controlled trials have shown that in- 
filtration of a local anesthetic (e.g., bupivacaine) into the extraperitoneal region or trocar incisions of patients reduces postoperative pain [6-8]. In several studies, a local anesthetic has been infiltrated into both the extraperitoneal region and trocar incisions, or only one of them [9, 10]. Based on current literature, we hypothesized that infiltration of bupivacaine into the preperitoneal space and trocar incisions of patients can reduce postoperative pain.

The present study aimed to compare the postoperative pain scores of patients in three groups. In patients of group I, local anesthetic was infiltrated into the preperitoneal space and trocar incisions, whereas in patients of group II, it was infiltrated only into the trocar incisions. No local anesthetic was administered to patients of group III.

\section{Material and methods}

Between March 2017 and February 2018, a total of 65 patients were included in this study. The patients who underwent repair of inguinal hernia between the ages of 17 to 70 years and those who belonged to the American Society of Anesthesiologists (ASA) class I and II were included in this study. Patients with bilateral inguinal and scrotal hernia, those who were previously operated for inguinal hernia and had an incision in the lower abdomen, those who had a history of alcohol and drug abuse, and those who had preoperative pain and strangulation signs were not included in this study.

After obtaining approval from the local ethics committee, a preliminary study was initially performed with five patients in each group. The sample size of the study was determined based on the results of the preliminary study. The standard effective size was determined to be 0.88 with $5 \%$ error and $80 \%$ power, and the sample size of $n=20$ was considered appropriate.

An internet-based random number generator was used for randomization (www.random.org). Preoperatively, group numbers I-III were randomly assigned and sent to the operating room in a closed envelope. Bupivacaine was administered in the final stage of the operation according to the group number received. In the case of group I, $50 \mathrm{mg}$ of $0.5 \%$ bupivacaine was infiltrated into the preperitoneal space and $10 \mathrm{mg}$ of bupivacaine was simultaneously injected into each trocar incision. In the case of group II, only $10 \mathrm{mg}$ of bupivacaine was injected into each trocar incision. However, no local anesthetic was administered in the case of group III.

Under general anesthesia, a $1 \mathrm{~cm}$ incision was made $1 \mathrm{~cm}$ inferior to the umbilical border. A 10$\mathrm{mm}$ trocar was placed in the preperitoneal space, and blunt dissection continued with a 30-degree camera. After the preperitoneal space was observed, two 5-mm working trocars were placed in the midline at $2 \mathrm{~cm}$ and $6 \mathrm{~cm}$ inferiorly from the umbilicus to the symphysis pubis. Bogros and Retzius areas were expanded with sharp and blunt dissections, respectively, and the Cooper ligament with cord structures was exposed. The hernia sac was freed from the anatomical structures of the cord. A $15 \times$ $13 \mathrm{~cm}$ Prolene mesh was spread over the preperitoneal area to cover all hernial defects. Prolene mesh was fixed to the Cooper ligament and the abdominal wall with the absorbable fixation device. At this stage, if the patient belonged to group I, $50 \mathrm{mg}$ of bupivacaine was instilled into the preperitoneal space, and the air was evacuated. Subsequently, $10 \mathrm{mg}$ of bupivacaine was injected into each trocar incision, and the skin was sutured. In the case of group II, the patient was only injected with $10 \mathrm{mg}$ of bupivacaine into each trocar incision. No local anesthetic was administered to the patients of group III. No intraoperative or postoperative complications were observed, and a drain was not used for any patient. All patients were operated on laparoscopically, and uneventfully discharged on the first postoperative day. No patient was re-operated.

Postoperative pain was assessed using the Visual Analog Scale (VAS) to digitize values that cannot be measured numerically. Typically, the extremes of the parameter to be evaluated are written on both ends of a 100-mm line. For example, no pain (1 on the scale) and the worst pain (10 on the scale) are written on either ends of a line, and the patient marks the current pain level on this line. The distance from the point of no pain to the patient's mark indicates the patient's pain level $[9,10]$. The VAS has been considered reliable for a long time and is accepted worldwide owing to its safety and ease of application $[9,10]$. In the current study, the patient was asked to draw or mark a line on the scale corresponding to their current pain level. Postoperative pain was assessed by a general practitioner who was blinded to randomization by VAS, but these scores were not reported to the surgeon. The following template can 
be used to determine the values on the line. In our study, we used a 10-unit visual scale.

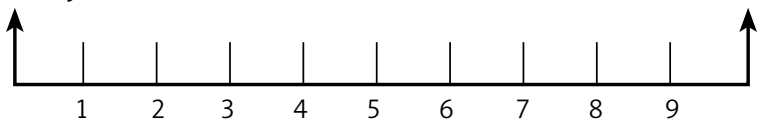

Postoperatively, prophylactic analgesic medications were not administered in any of the three groups. However, patients who presented with symptomatic pain and those who requested analgesia were administered with an intravenous infusion of $1 \mathrm{~g}$ of paracetamol, and the total dosage was noted.

\section{Ethics}

Written informed consent was obtained from all patients included in this study. The research was conducted according to the principles of the World Medical Association Declaration of Helsinki "Ethical Principles for Medical Research Involving Human Subjects." The approval of a local ethics committee was obtained.

\section{Statistical analysis}

Descriptive statistical methods including mean, standard deviation, frequency, and ratio were used to evaluate the data. The Kolmogorov-Smirnov test was used to analyze the distribution of variables, the independent samples $t$-test and Mann-Whitney $U$ test were used to analyze quantitative data, and the $\chi^{2}$ test was used to analyze qualitative data. Spearman correlation and logistic regression analyses were used for multivariate analysis. Statistical analysis was performed using the SPSS software package for Windows (SPSS version 17.0, SPSS Inc., Chicago, Illinois, USA).

\section{Results}

The patients were divided into three groups. A local anesthetic (bupivacaine) was infiltrated into the preperitoneal space and trocar incisions of the 23 patients in group I, whereas it was infiltrated only into the trocar incisions of the 21 patients in group II.

Table I. Demographic characteristics of patients

\begin{tabular}{|c|c|c|c|c|c|c|c|}
\hline \multirow[t]{2}{*}{ Parameter } & \multicolumn{2}{|c|}{ Group I } & \multicolumn{2}{|c|}{ Group II } & \multicolumn{2}{|c|}{ Group III } & \multirow[t]{2}{*}{$P$-value } \\
\hline & $\begin{array}{c}\text { Mean } \pm \text { SD } \\
\text { or } n(\%)\end{array}$ & Median & $\begin{array}{c}\text { Mean } \pm \text { SD } \\
\text { or } n(\%)\end{array}$ & Median & $\begin{array}{l}\text { Mean } \pm \text { SD } \\
\text { or } n(\%)\end{array}$ & Median & \\
\hline Age [years] & $54.5 \pm 13.7$ & 56.0 & $49.0 \pm 13.9$ & 50.0 & $59.2 \pm 11.2$ & 60.0 & $0.052^{\mathrm{A}}$ \\
\hline \multicolumn{8}{|l|}{ Sex: } \\
\hline Male & $22(95.7)$ & & $20(90.5)$ & & $21(100.0)$ & & \multirow[t]{2}{*}{$>0.05^{x^{2}}$} \\
\hline Female & $1(4.3)$ & & $1(9.5)$ & & $0(0.0)$ & & \\
\hline Height & $170.8 \pm 7.1$ & 170.0 & $170.9 \pm 6.0$ & 170.0 & $170.9 \pm 5.1$ & 170.0 & $0.994^{K}$ \\
\hline Weight & $75.7 \pm 10.7$ & 75.0 & $74.1 \pm 12.6$ & 78.0 & $79.7 \pm 8.2$ & 80.0 & $0.441^{\mathrm{K}}$ \\
\hline $\mathrm{BMI}$ & $26.1 \pm 3.6$ & 26.4 & $25.0 \pm 3.5$ & 25.5 & $27.3 \pm 2.9$ & 27.0 & $0.165^{\mathrm{K}}$ \\
\hline \multicolumn{8}{|l|}{ ASA score: } \\
\hline I & $16(69.6)$ & & $15(71.4)$ & & $10(47.6)$ & & \multirow[t]{2}{*}{$0.202^{x^{2}}$} \\
\hline$\|$ & $7(30.4)$ & & $6(28.6)$ & & $11(52.4)$ & & \\
\hline \multicolumn{8}{|c|}{ Previous surgical history: } \\
\hline- & 21 (91.3) & & $19(90.5)$ & & $21(100.0)$ & & \multirow[t]{2}{*}{$0.359^{x^{2}}$} \\
\hline+ & $2(8.7)$ & & $2(9.5)$ & & $0(0.0)$ & & \\
\hline \multicolumn{8}{|l|}{ Smoking: } \\
\hline- & $14(60.9)$ & & $13(61.9)$ & & $13(61.9)$ & & \multirow[t]{2}{*}{$0.997^{x^{2}}$} \\
\hline+ & $9(39.1)$ & & $8(38.1)$ & & $8(38.1)$ & & \\
\hline
\end{tabular}

${ }^{A}$ Anova; ${ }^{K}$ Kruskal-Wallis; ${ }^{2}$ chi-square test. 
The 21 patients in group III were not administered with any local anesthetic. There was no significant difference regarding age, gender, body mass index, ASA class, history of previous abdominal surgery, or smoking between the three groups $(p>0.05)$ (Table I). VAS score at $4 \mathrm{~h}$ was significantly higher in group III than in groups I and II ( $p<0.05)$, with no significant difference between groups $\mid$ and $\|(p>$ $0.05)$. VAS score at $24 \mathrm{~h}$ did not significantly differ between the three groups $(p>0.05)$ (Figure 1$)$. The analgesic medication and dosage were significantly higher in group III than in groups I and II $(p<0.05)$ (Figures 2, 3), with no significant difference between groups I and II ( $p>0.05)$ (Table II). The length of hospitalization was one day for all three groups.

\section{Discussion}

Although minimally invasive surgery is associated with reduced postoperative pain, it is not entirely painless. Patients undergoing laparoscopic repair of

\section{A}

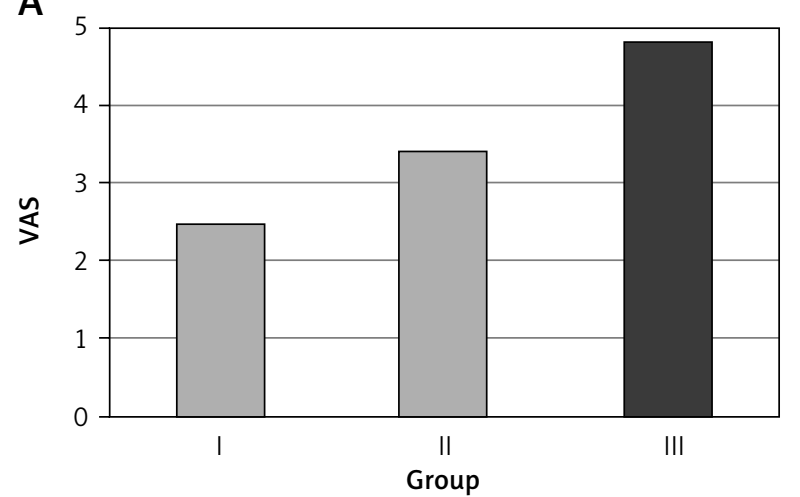

Figure 1. Postoperative VAS score at $4^{\text {th }}(A)$ and $24^{\text {th }} \mathrm{h}(\mathrm{B})$

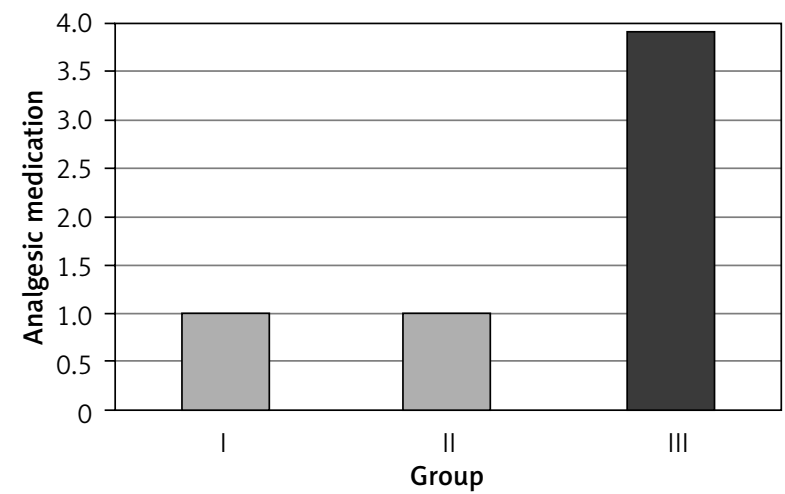

Figure 2. Dosage of analgesic medication inguinal hernia might experience postoperative pain requiring narcotic analgesic medications. Postoperative pain control reduces the need for analgesic medications, postoperative complications, length of hospitalization, and expenditure [11, 12]. In our study, we observed greater postoperative pain relief in patients who received a local anesthetic. Several studies in the literature have revealed that preperitoneal local anesthesia is effective in pain control.

Since TEP repair provides a limited preperitoneal area, it allows for easy and effective application of a local anesthetic [7, 13, 14]. A randomized controlled trial conducted by Bar-Dayan et al., including 44 patients divided into two groups, observed that pain was significantly lower in the group that received preperitoneal infiltration of bupivacaine than in the control group [13]. Another randomized controlled trial conducted by Hon et al. revealed that $0.5 \%$ bupivacaine instilled into the preperitoneal space of patients undergoing TEP repair resulted in effective pain control compared with the control group

B

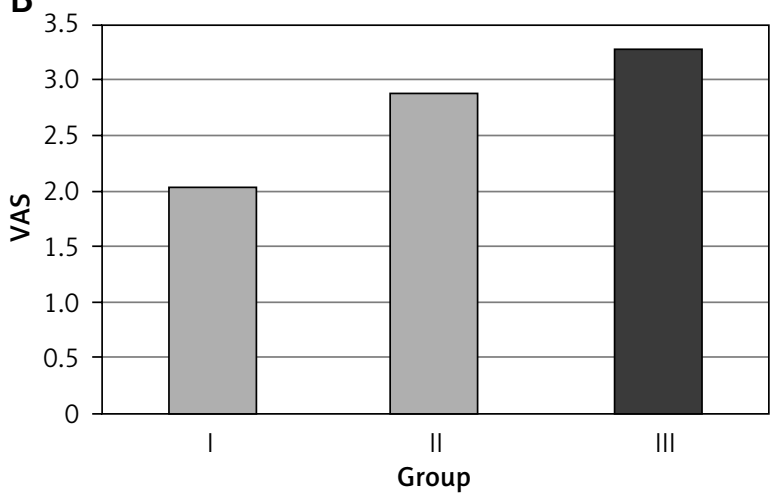

(B)

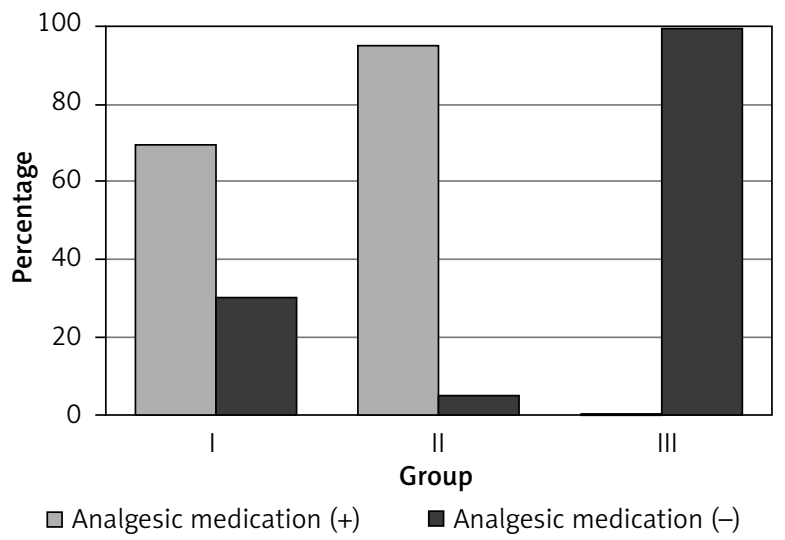

Figure 3. Comparison of dosages of analgesic medications between groups 
Table II. VAS score and dosage of analgesic medication

\begin{tabular}{|c|c|c|c|c|c|c|c|}
\hline \multirow[t]{2}{*}{ Parameter } & \multicolumn{2}{|c|}{ Group I } & \multicolumn{2}{|c|}{ Group II } & \multicolumn{2}{|c|}{ Group III } & \multirow[t]{2}{*}{$P$-value } \\
\hline & $\begin{array}{c}\text { Mean } \pm \text { SD } \\
\text { or } n(\%)\end{array}$ & Median & $\begin{array}{c}\text { Mean } \pm \text { SD } \\
\text { or } n(\%)\end{array}$ & Median & $\begin{array}{c}\text { Mean } \pm \text { SD } \\
\text { or } n(\%)\end{array}$ & Median & \\
\hline VAS at $4 \mathrm{~h}$ & $2.5 \pm 2.1$ & & $3.4 \pm 2.7^{\star}$ & 4.0 & $4.8 \pm 2.3$ & 4.0 & $0.010^{k}$ \\
\hline VAS at $24 \mathrm{~h}$ & $2.0 \pm 2.1$ & 1.0 & $2.9 \pm 2.4$ & 3.0 & $3.3 \pm 2.3$ & 3.0 & $0.161^{\mathrm{K}}$ \\
\hline \multicolumn{8}{|c|}{ Analgesic medication: } \\
\hline- & $16(69.6)^{\star}$ & & $16(76.1)$ & & $0(0.0)$ & & \multirow[t]{2}{*}{$<0.001^{x^{2}}$} \\
\hline+ & $7(30.4)$ & & $5(23.9)^{*}$ & & $21(100.0)$ & & \\
\hline Analgesic dosage & $1.0 \pm 2.1^{*}$ & 0.0 & $1.0 \pm 1.8^{\star}$ & 0.0 & $3.9 \pm 0.9$ & 4.0 & $<0.001^{K}$ \\
\hline
\end{tabular}

${ }^{K}$ Kruskal-Wallis (Mann-Whitney $U$ test); ${ }^{x^{2}}$ Chi-square test. * Difference with group III $p<0.005$.

[14]. A randomized controlled trial was conducted by O'Riordain et al., including 56 patients who were divided into two groups and compared based on their pain scores; one group received pre-peritoneal bupivacaine $(n=29)$ and the other received saline treatment $(n=27)$. The study results revealed that the pain score of the patients who received bupivacaine was statistically significantly lower. Although this difference was observed at $24 \mathrm{~h}$, the patients who received bupivacaine recovered faster and returned to normal activity earlier.

In contrast, some studies, such as the randomized controlled trial conducted by Abbas et al., have shown that infiltration of bupivacaine into the pre-peritoneal space did not provide significant postoperative pain relief [8]. In a randomized controlled trial conducted by Saff et al., including 42 patients, $60 \mathrm{ml}$ of $0.125 \%$ bupivacaine was infiltrated into the pre-peritoneal space, and no significant difference regarding the postoperative pain scores, need for analgesic medication, or length of hospitalization was observed between the patients who received bupivacaine and those who were in the control group [6]. Dath et al. found no significant difference regarding the pain scores and need for narcotic analgesic medications between the patients in whom trocar incisions were infiltrated with $20 \mathrm{mg}$ of $0.5 \%$ bupivacaine and epinephrine during laparoscopic cholecystectomy and the control group [15]. A similar study conducted by Hasaniya et al. infiltrated $0.5 \%$ bupivacaine into trocar incisions during laparoscopic cholecystectomy. In this study, the patients who were administered with a local anesthetic experience cost-effective pain control because of the reduced need for postoperative analgesic and antiemetic medications [16].
In contrast, in a systematic review and metaanalysis conducted by Tong et al., they reviewed eight RCTs that included a total of 373 patients and investigated the outcomes of extraperitoneal bupivacaine analgesia versus control in laparoscopic TEP hernia repair. They found that extraperitoneal bupivacaine treatment during laparoscopic TEP inguinal hernioplasty is not more efficacious for the reduction of postoperative pain than placebo and they do not recommend extraperitoneal bupivacaine analgesia during laparoscopic TEP inguinal hernia repair for routine practice [17].

Previous studies have validated VAS to be a consistent and valid scale for the quantitative measurement of subjective perceptions of pain and anxiety $[18,19]$. Additionally, VAS can be used as a numerical value that can be linearly increased and rated to evaluate pain severity [20].

However, VAS scoring reflects the patient's perception of pain rather than its objective severity, and it would be more appropriate to prefer other scoring systems, especially in elderly patients $[9,10]$. In addition to VAS, we believe that other scoring systems, such as the Numeric Rating Scale or Verbal Descriptor Scales, can improve the reliability of data by decreasing pain-scale errors.

According to guidelines for laparoscopic (TAPP) and endoscopic (TEP) treatment of inguinal hernia, published by the International Endohernia Society (IEHS) in 2011, "Additional use of local anaesthetic positively influences postoperative pain in TEP and TAPP. Infiltration of trocar wounds with long-acting local anaesthetic in TAPP improves patient's well-being and accelerates return to ambulation (Grade D). To improve postoperative pain control, trocar wounds 
can be infiltrated by local anesthetics. Such effect was not reported in TAPP yet, although routine infiltration of the wound after hernia repair provides extra pain control and limits the use of analgesics" [21].

A study that compared inguinal hernia surgery under spinal and general anesthesia revealed that VAS scores were significantly lower in the spinal anesthesia group at 1,6 , and $12 \mathrm{~h}$ postoperatively compared to the general anesthesia group ( $p=$ $0.001,0.001$, and 0.011 , respectively). Although the VAS score was lower in the spinal anesthesia group than in the general anesthesia group at $24 \mathrm{~h}$ postoperatively, the difference was not statistically significant $(p=0.643)$ [22].

Laparoscopic techniques minimize the risk of soft tissue and nerve trauma in the groin region, allow quick return to work and reduce the level of postoperative pain. Therefore, younger patients may benefit most from laparoscopic surgery [21, 23].

Yildirim et al. conducted another study using slit and nonslit mesh (SM and NSM) for the repair of inguinal hernia for the diameter less than $5 \mathrm{~cm}$ and evaluated early postoperative pain score by VAS; the pain levels of the NSM group were statistically significantly lower than those of the SM group in the first, $6^{\text {th }}, 12^{\text {th }}$, and $24^{\text {th }}$ h postoperatively $(p<0.001$, $p<0.001, p<0.001$, and $p=0.004)$. When the chronic pain was investigated at the $3^{\text {rd }}$ month, the SM group had a significantly higher pain level than that of the NSM group $(p=0.004)$. When the hernia diameter was larger than $5 \mathrm{~cm}$, there was no significant difference between the groups in the early postoperative period of pain [24].

The other method for reducing postoperative pain in patients who have undergone laparoscopic inguinal hernia repair is fixation of mesh with glue $[25,26]$. A prospective trial that included 146 patients who underwent the laparoscopic TAPP method for inguinal hernia repair with fixation of mesh by glue showed that the frequency and intensity of the pain after surgery were significantly decreased compared to that experienced preoperatively, which is especially important for younger and professionally active patients [27].

In another study conducted by Mitura et al., they found that preoperative intensity of pain was significantly higher in patients aged $<40$ years $(63.7 \%$; VAS 5.4 ) than in patients $>60$ years $(54.3 \%$; VAS 4.8$)$ $(p=0.008)$ and assessment of pain may help determine the group of younger patients who could bene- fit the most from inguinal hernia repair in the preoperative period. This study showed that longer hernia duration prior to surgery causes increased pre- and postoperative pain [28].

Unlike several studies in the literature, our study involved three groups. We aimed to obtain additional information on the etiology of pain by infiltration of a local anesthetic into the preperitoneal space and trocar incisions in group I and only into the trocar incisions in group II. Although there was no significant difference in the pain scores between these two groups, there was a quantitative difference. Further studies are needed to clarify this aspect.

Notably, an injection of a long-acting local anesthetic into the preperitoneal space and trocar incisions during TEP repair reduces early postoperative pain, ultimately reducing the dosage of postoperative analgesic medication. Moreover, infiltration of bupivacaine into the preperitoneal space is easy, reliable, and effective. Therefore, local anesthesia with infiltration of bupivacaine into the preperitoneal space and trocar incisions during laparoscopic repair of inguinal hernia can be safely considered to provide comfort in the early postoperative period. We found no significant difference between the groups regarding length of hospitalization. There is also a need for further studies to definite the effectiveness of early discharge.

\section{Conclusions}

Infiltration of a local anesthetic into the preperitoneal space and trocar incisions was effective in reducing pain in the first $4 \mathrm{~h}$ after surgery, but not in the long term. Therefore, we suggest the use of preperitoneal local anesthesia with bupivacaine in the laparoscopic repair of inguinal hernia.

\section{Conflict of interest}

The authors declare no conflict of interest.

\section{References}

1. Rosenberg J, Bisgaard T, Kehlet H, et al. Danish Hernia Database recommendations for the management of inguinal and femoral hernia in adults. Dan Med Bull 2011; 58: C4243.

2. Bay-Nielsen M, Kehlet H, Strand L, et al. Quality assessment of 26304 herniorrhaphies in Denmark: a prospective nationwide study. Lancet 2001; 358: 1124-8.

3. Stoker DL, Spiegelhalter DJ, Singh R, et al. Laparoscopic versus open inguinal hernia repair: randomised prospective trial. Lancet 1994; 343: 1243-45. 
4. Wellwood J, Sculpher MJ, Stoker D, et al. Randomised controlled trial of laparoscopic versus open mesh repair for inguinal hernia: outcome and cost. BMJ 1998; 317: 103-10.

5. Chan KL, Hui WC, Tam PK. Prospective randomized single center, single-blind comparison of laparoscopic vs open repair of pediatric inguinal hernia. Surg Endosc 2005; 19: 927-32.

6. Saff GN, Marks RA, Kuroda M, et al. Analgesic effect of bupivacaine on extraperitoneal laparoscopic hernia repair. Anesth Analg 1998; 87: 377-81.

7. O'Riordain DS, Kelly P, Horgan PG, et al. A randomized controlled trial of extraperitoneal bupivacaine analgesia in laparoscopic hernia repair. Am J Surg 1998; 176: 254-7.

8. Abbas MH, Hamade A, Choudhry MN, et al. Infiltration of wounds and extraperitoneal space with local anesthetic in patients undergoing laparoscopic totally extraperitoneal repair of unilateral inguinal hernias: a randomized double blind placebo controlled trial. Scand J Surg 2010; 99: 18-23.

9. Clark WC, Yang JC, Tsui SL, et al. Unidimensional pain rating scales: a multidimensional affect and pain survey (MAPS) analysis of what they really measure. Pain 2002; 98: 241-7.

10. Gagliese L, Weizblit N, Ellis W, et al. The measurement of postoperative pain: a comparison of intensity scales in younger and older surgical patients. Pain 2005; 117: 412-20.

11. Bays A, Barry L, Vasilenko P. The use of bupivacaine in elective inguinal herniorrhaphy as a fast and safe technique for relief of postoperative pain. Surg Gynecol Obstet 1991; 173: 433-7.

12. Edelman SD, Misiakos PF, Moses K. Extraperitoneal laparoscopic hernia repair with local anesthesia. Surg Endosc 2001; 15: 976-80.

13. Bar-Dayan A, Natour M, Bar-Zakai B, et al. Preperitoneal bupivacaine attenuates pain following laparoscopic inguinal hernia repair. Surg Endosc Other Interv Tech 2004; 18: 1079-81.

14. Hon SF, Poon CM, Leong HT, et al. Pre-emptive infiltration of bupivacaine in laparoscopic total extraperitoneal hernioplasty: a randomized controlled trial. Hernia 2009; 13: 53-6.

15. Dath D, Park AE. Randomized, controlled trial of bupivacaine injection to decrease pain after laparoscopic cholecystectomy. Canadian J Surg 1999; 42: 284-8.

16. Hasaniya NW, Zayed FF, Faiz H, et al. Preinsertion local anesthesia at the trocar site improves perioperative pain and decreases costs of laparoscopic cholecystectomy. Surg Endosc 2001; 15: 962-4.

17. Tong YS, Wu CC, Bai CH, et al. Effect of extraperitoneal bupivacaine analgesia in laparoscopic inguinal hernia repair: a meta-analysis of randomized controlled trials. Hernia 2014; 18: 177-83.

18. Facco E, Stellini E, Bacci C, et al. Validation of visual analogue scale for anxiety (VAS-A) in preanesthesia evaluation. Minerva Anestesiol 2013; 79: 1389-95.

19. Brokelman RB, Haverkamp D, van Loon C, et al. The validation of the visual analogue scale for patient satisfaction after total hip arthroplasty. Eur Orthop Traumatol 2012; 3: 101-5.

20. Myles PS, Troedel S, Boquest M, et al. The pain visual analog scale: is it linear or nonlinear? Anesth Analg 1999; 89: 1517-20.

21. Bittner R, Arregui ME, Bisgaard T, et al. Guidelines for laparoscopic (TAPP) and endoscopic (TEP) treatment of inguinal hernia [International Endohernia Society (IEHS)]. Surg Endosc 2011; 25: 2773-843.
22. Yildirim D, Hut $A$, Uzman $S$, et al. Spinal anesthesia is safe in laparoscopic total extraperitoneal inguinal hernia repair. A retrospective clinical trial. Videosurgery Miniinv 2017; 12: 417-27.

23. Bansal VK, Misra MC, Babu D, et al. A prospective, randomized comparison of long-term outcomes: chronic groin pain and quality of life following totally extraperitoneal (TEP) and transabdominal preperitoneal (TAPP) laparoscopic inguinal hernia repair. Surg Endosc 2013; 27: 2373-82.

24. Yildirim D, Donmez T, Ozcevik H, et al. Comparison of slit mesh versus nonslit mesh in laparoscopic extraperitoneal hernia repair. Videosurgery Miniinv 2018; 13: 469-76.

25. Kaul A, Hutfless S, Le H, et al. Staple versus fibrin glue fixation in laparoscopic total extraperitoneal repair of inguinal hernia: a systematic review and meta-analysis. Surg Endosc 2012; 26: 1269-78.

26. Shah NS, Fullwood C, Siriwardena AK, et al. Mesh fixation at laparoscopic inguinal hernia repair: a meta-analysis comparing tissue glue and tack fixation. World J Surg 2014; 38: 2558-70.

27. Mitura K, Garnysz K, Wyrzykowska D, et al. The change in groin pain perception after transabdominal preperitoneal inguinal hernia repair with glue fixation: a prospective trial of a single surgeon's experience. Surg Endosc 2018; 32: 4284-9.

28. Mitura K, Śmietański M, Kozieł S, et al. Factors influencing inguinal hernia symptoms and preoperative evaluation of symptoms by patients: results of a prospective study including 1647 patients. Hernia 2018; 22: 585-91.

Received: 27.01.2019, accepted: 10.03.2019. 